\title{
Salt Tolerance Potential in Orchid Oncidium hybridum Plants Revealed by Halophenotyping and Genotyping
}

ISSN: 2637-7659

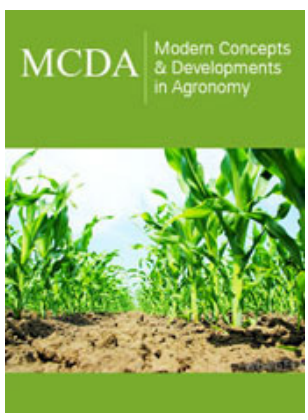

*Corresponding author: Zhongxiong Lai, Institute of Horticultural Biotechnology, Fujian Agriculture and Forestry University, Fuzhou, China

$\mathrm{Xu}$ XuHan, Institute of Horticultural Biotechnology, Fujian Agriculture and Forestry University, Fuzhou, China

Submission: 眥 November 13, 2020

Published: 眥December 02, 2020

Volume 7 - Issue 4

How to cite this article: Jing Zhang, Shanshan Wang, Jingjie Ban, ZhongxiongLai, Xu XuHan, et al. Salt Tolerance Potential in Orchid Oncidium hybridum Plants Revealed by Halophenotyping and Genotyping. Mod Concep Dev Agrono. 7(4). MCDA. 000669. 2020. DOI: 10.31031/MCDA.2020.07.000669

Copyright@ Zhongxiong Lai, Xu XuHan, This article is distributed under the terms of the Creative Commons Attribution 4.0 International License, which permits unrestricted use and redistribution provided that the original author and source are credited.

\begin{abstract}
Jing Zhang ${ }^{1}$, Shanshan Wang ${ }^{1}$, Jingjie Ban ${ }^{1}$, Shuai Fu ${ }^{1}$, Zhengchun Linn ${ }^{1}$, Xu Shen ${ }^{1}$, Wei Ye ${ }^{2}$, Kexuan Zhang ${ }^{3}$, Zhikang Jiang ${ }^{3}$, Kai Wun Yeh", Yukun Chen ${ }^{1}$, Yuling Lin ${ }^{1}$, Zhongxiong Lai ${ }^{1 *}$, and Xu XuHan ${ }^{1,3,5 *}$
\end{abstract}

${ }^{1}$ Institute of Horticultural Biotechnology, Fujian Agriculture and Forestry University, Fuzhou, China

${ }^{2}$ Sanming Academy of Agricultural Sciences, Sanming, Fujian, China

${ }_{3}^{3}$ Jinan Phyto RTD Eco-Engineering Co. Ltd., Jinan, Shandong, China

${ }^{4}$ Institute of Plant Biology, Taiwan University, Taipei, China

${ }^{5}$ Institute de la Recherche Interdisciplinairede Toulouse, IRIT-ARI, Toulouse, France

\begin{abstract}
Orchid plants are thought to be glycophytes. Oncidium hybridum var. Gower Ramsey (OhGR) plants were tested for the halotolerance by cultivation in $0-5000 \mathrm{mM} \mathrm{NaCl}$ solutions. Phenotypes such as leaf morphology and electrical conductivity of the salt treated plants for a week, and gene expression spectrums of different parts of those plants were analyzed. Electron transfer carrier genes OhFNR and OhFD as an example of house-keeping genes, a transcription factor OhMYB1R1and a phospholipase D gene OhPLD 1 as tolerance responsive genes, were investigated with $\mathrm{qPCR}$. It was found that OhGR plants absorbed salt without secretion, the leaves turned yellow in the salt treatment with a basal to upper manner and the electrical conductivity of the leave tissues increased accordingly with the leaf morphological change. The genes assessed showed stress timing and tissue specific differential spectrums in which OhFD-OhF$N R$ were co-inhibited by salt stress, OhMYB1R1 and OhPLDs were stress inducible and enhanced. At the meanwhile, the upper leaves, the pseudobulbs and the roots kept morphologically normal and alive in most tested OhGR plants in various salt conditions tested, even a high amount of salt had been accumulated in the plants as revealed by electroconductivity measures. Our results show that OhGR plants have high salt tolerance potential based on their ordered cell and molecular mechanisms.
\end{abstract}

Keywords: Halotolerance; Halogenomics; Ferredoxin; Phospholipase D; MYB

\section{Introduction}

Stress tolerance is one of the hot issues in agriculture and horticulture [1,2]. Orchids, the biggest family Orchidaceae in angiosperms, including more than 700 genera, and more than 750 species in Oncidium [3], are thought to be glycophytes. Many orchid plants e.g. Oncidium hybridum, live far away from seawater, and can even live on the bark of trees. Halophytic and salt-tolerant orchids were rear recorded. However, orchids cultivatable in salinity remain to be explored in plant breeding. In the present study, an orchid variety 0 . hybridum var. Gower Ramsey originated from and cultivated domestically in non-saline environment was employed to test its salt stress responses. To our suppress, a high degree of salt tolerance was encountered.

\section{Objective}

The objective of the present work is to explore salt stress responses and salt tolerance potential of 0 . hybridum via phenotyping and genotyping.

\section{Material and Methods}

Oncidium hybridum var. Gower Ramsey plants (OhGR Plants) cultivated in soil pots in Fuzhou were transferred to sodium chloride solutions, with a $16 \mathrm{~h}$ photoperiod at $25^{\circ} \mathrm{C}$, for 1-2 weeks. Sodium chloride concentrations were arranged as $0,200,400,600,800,1000$, 2000, 3000, 4000, 5000mM, 3 plants in each treated group, 3 repeats. To prevent cloning 
effect, other 0 . hybridum var. Gower Ramsey plants cultivated in other nurseries, and plants of another variety Kutoo of Oncidium were also salt treated and observed. The sampled plants were transplanted to soil pots for further observations.

Leaf morphology was analyzed with microscopy and electric conductivity were measured by electric conductivity testers (Asmik, Hangzhou Asmik Sensor Technology Co. Ltd.). Different leaf tissues of different morphological characteristics were sampled for electric conductivity measure and RNA isolation. Total RNA was isolated according to manufacturer's protocol for RNA extraction kids, from control ( $0 \mathrm{mM} \mathrm{NaCl})$ leaf tissues and different salt-treated leaf parts, i.e. yellow vs. green, or upper vs. basal, at different time lengths during 2 weeks.

For quantitative real time PCR (qPCR), the ferredoxin OhFD [4] and its coupled electron carrier gene OhFNR (KX461908.1) [5], an
MYB transcription factor OhMYB1R1 cloned by Li et al. [4], and the cDNA sequence of phospholipase D gene OhPLD 1 was obtained from OhGR transcriptome data, and the primers of the cDNAs were designed based on the cDNA sequences. The qPCR was performed according to Li et al. [4].

\section{Result}

\section{Morphological phenotyping}

OhGR Plants showed normal development in the investigated population in the present work (Figure 1.1), and at younger stage, 2-3-year age, it showed a typical 4-leaf patten in which 2 leaves positioned near the apical part, 2 leaves near the basal part (Figure 1A). The 4-leaf stage kept stable for months before it turned to multi-leaf and flowering stages (Figure 1B) in our cultivation condition and was proper for our phenotyping analysis.

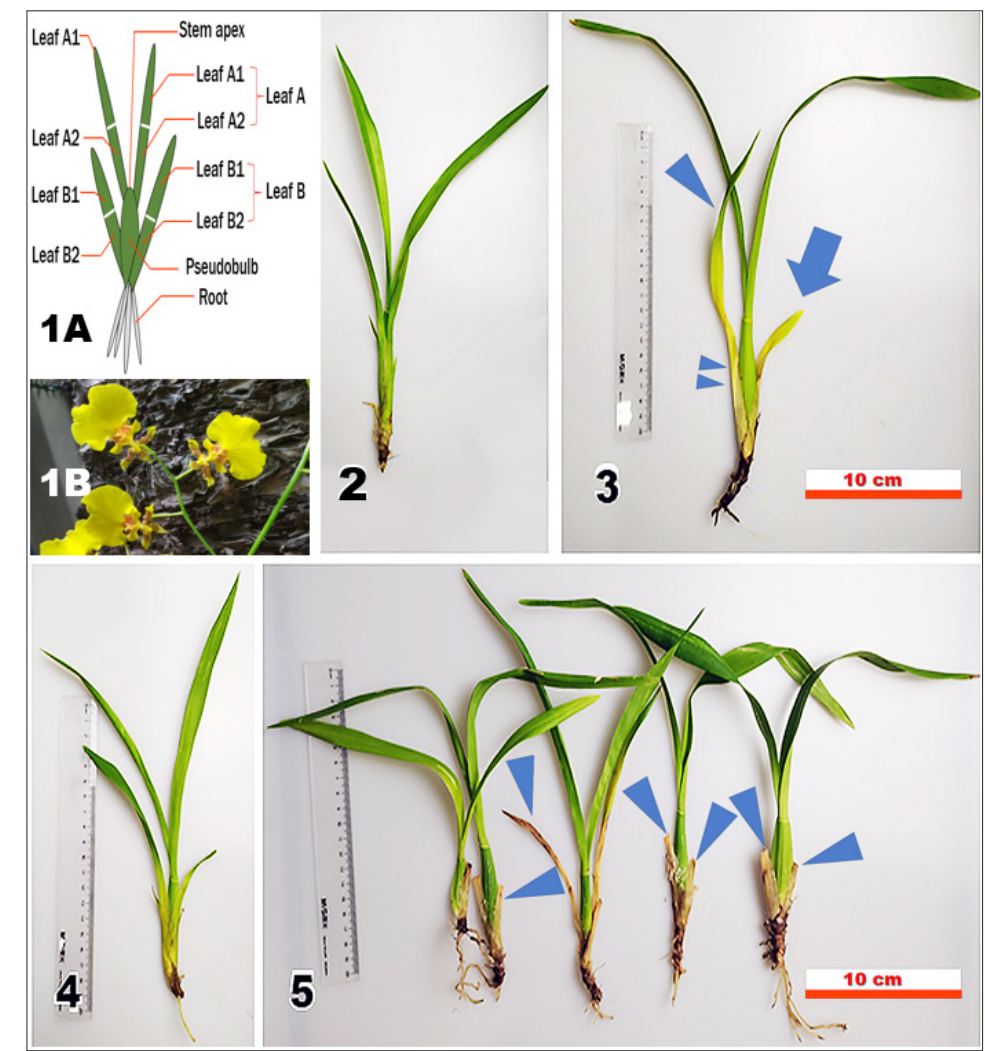

Figure 1: Morphological changes of $\mathrm{NaCl}$ solution treated OhGR plants.

1A: Graphic representation of the 4-leaf developmental stage. Leaves A1 and A2 represent the upper and basal parts of the upper leaves and leaves B1and B2 represent the upper and basal parts of the basal leaves, respectively.

respectively.

1B: Flowers.

2: Plant of the control group, $0 \mathrm{mmol} / \mathrm{L}[\mathrm{NaCl}], 7 \mathrm{dpt}$.

3: Plant treated in $1000 \mathrm{mmol} / \mathrm{L}$ [ $\mathrm{NaCl}$, $7 \mathrm{dpt}$. Not the entire yellow basal leaf (arrow) and the yellow basal part (double arrowheads) with green upper part (single arrowhead) of the other basal leaf.

4: Plant treated in $1000 \mathrm{mmol} / \mathrm{L}[\mathrm{NaCl}]$, $7 \mathrm{dpt}$. Note the green basal leaves.

5: Plants pre-treated with $200,400,600,800,1000 \mathrm{mmol} / \mathrm{L}$ [ $\mathrm{NaCl}$ ] solutions (from left to right, respectively) for a week and then transplanted to $1000 \mathrm{mmol} / \mathrm{L}[\mathrm{NaCl}]$ solution, $7 \mathrm{dpt}$. Note the dead basal leaves (arrowheads) and upper green (in general) leaves. 2-5: bar $=10 \mathrm{~cm}$. 
Within 7 days post-treatment (dpt), the OhGR plants treated with series of $[\mathrm{NaCl}]$ solutions showed normal morphology at low concentration $(200 \mathrm{mmol} / \mathrm{L})$ compared with the control group (Figure 1.2). When in higher [ $\mathrm{NaCl}]$ solutions, i.e. 400, 600 and $800 \mathrm{mmol} / \mathrm{L}$, some OhGR plants exhibited slight yellowish in the basal leaves, while the upper leaves were still green. When treated with $1000 \mathrm{mmol} / \mathrm{L}[\mathrm{NaCl}]$ solution, the upper leaves still kept green whereas clear yellowing of the basal leaves was expressed (Figure 1.3), though all leaves remained green in a few OhGR plants (Figure 1.4).

To verify if the salt tolerance was able to be induced by pretreatment with low $[\mathrm{NaCl}]$ solutions, OhGR plants were first treated with series of [ $\mathrm{NaCl}]$ solutions at $200,400,600,800,1000 \mathrm{mmol} / \mathrm{L}$ for a week, and then were transplanted in $1000 \mathrm{mmol} / \mathrm{L}[\mathrm{NaCl}]$ solution, and all the basal leaves of the pre-treated OhGR plants showed severe yellowing and dead, though the upper leaves kept green only showing yellowing in part (Figure 1.5). No salt pre- treatment was found helpful to increase salt tolerance, instead, accumulative effects were clear (Figure 1.5).

The morphology of the salt stressed OhGR plant leaves showed yellowing starting from the basal leaves, and particularly from the basal part of the basal leaves (Figure 1.3). The severe stressed OhGR plant showed basal leaf yellowing and death (Figure $1.3 \& 1.5$ ). The upper leaves exhibited limited yellowing areas in a dispersed distribution patten in severe stress conditions (Figure 1.5).

In the other variety, Kutoo plants treated with $1000 \mathrm{mmol} / \mathrm{L}$ $[\mathrm{NaCl}]$ solutions, showed similar phenotypic characters.

\section{Physiological phenotyping}

Electroconductivity of OhGR plants were examined to verify any absorption of $\mathrm{NaCl}$ in the plant tissues. As the $\mathrm{NaCl}$ concentration increased the plant tissues showed increased electroconductivity (Figure 2).

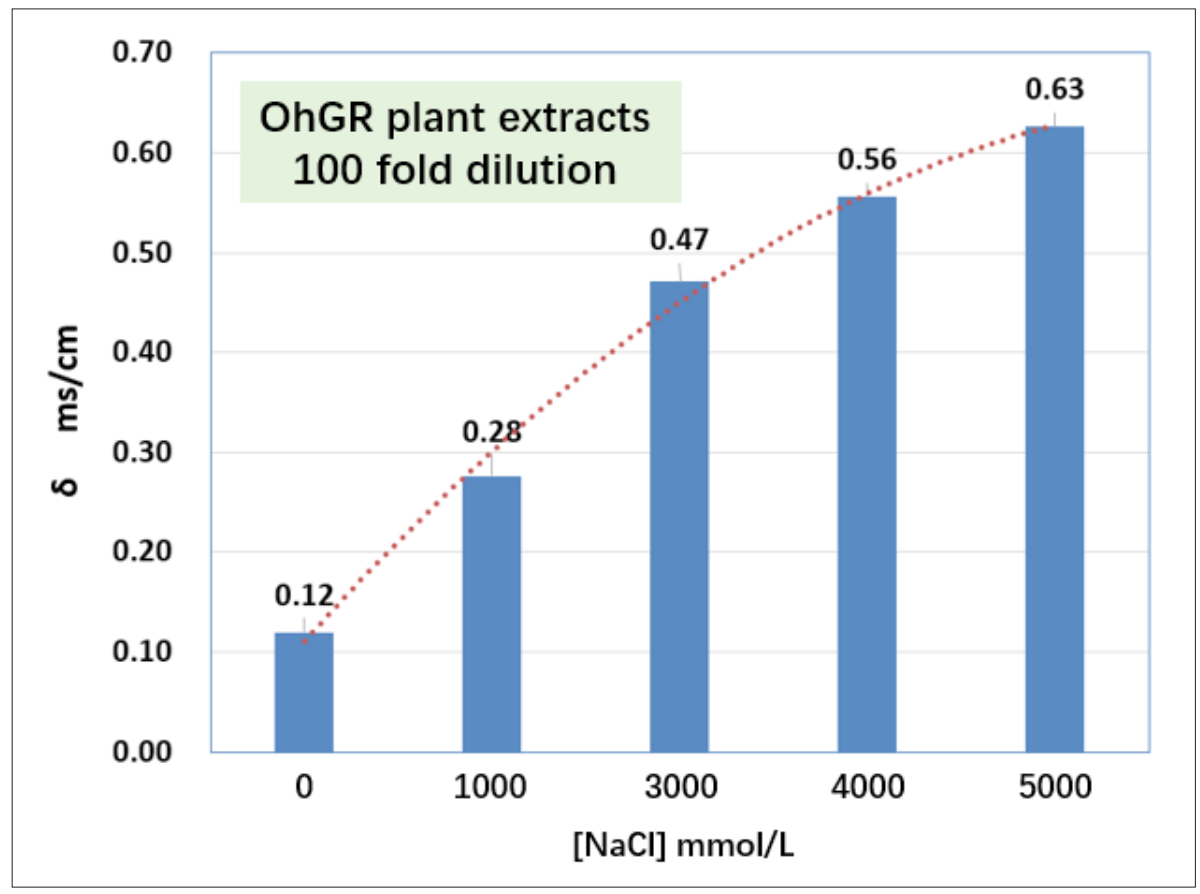

Figure 2: Electric conductivity $(\delta)$ of $\mathrm{NaCl}$ solution treated OhGR plants compared with irrigation water ([Na$\mathrm{Cl}]=0 \mathrm{mmol} / \mathrm{L}$ ) incubation condition, $5 \mathrm{dpt}$. The plant extracts were measured after 100 -fold dilution in water. Note: all the measured plants kept alive on $5 \mathrm{dpt}$.

\section{Genotyping}

Gene expressions of OhPLD 1, OhMYB1R1, OhFNR and OhFD of the OhGR plants treated with 1000,3000 and $5000 \mathrm{mmol} / \mathrm{L}[\mathrm{NaCl}]$ solutions for 5 days ( $5 \mathrm{dpt}$ ) were compared with the control plants. In each plant, the upper and basal leaves were separately checked and, in each leaf the upper part and the basal part were also examined separately (Figure 3). In general, the upper leaves (Figure 3: $1 \mathrm{~A}-4 \mathrm{~A}$ ), which kept green, exhibited very limited variations of the gene expressions compared with the control group ([NaCl] $0 \mathrm{mM}$ ). However, the basal leaves (Figure 3: 1B-4B), which turned yellow and most of them dead, showed significant differences. The OhPLD 1 and OhMYB1R1 enhanced the expressions as the salt concentration increased, whereas the OhFD the OhGR exhibited lowered expressions with the decrease of the salt concentration. The upper part of the basal leaves (leaves B1) presented differential expression differences from that of the basal part (leaves B2) (Figure 3: 1B-4B). 


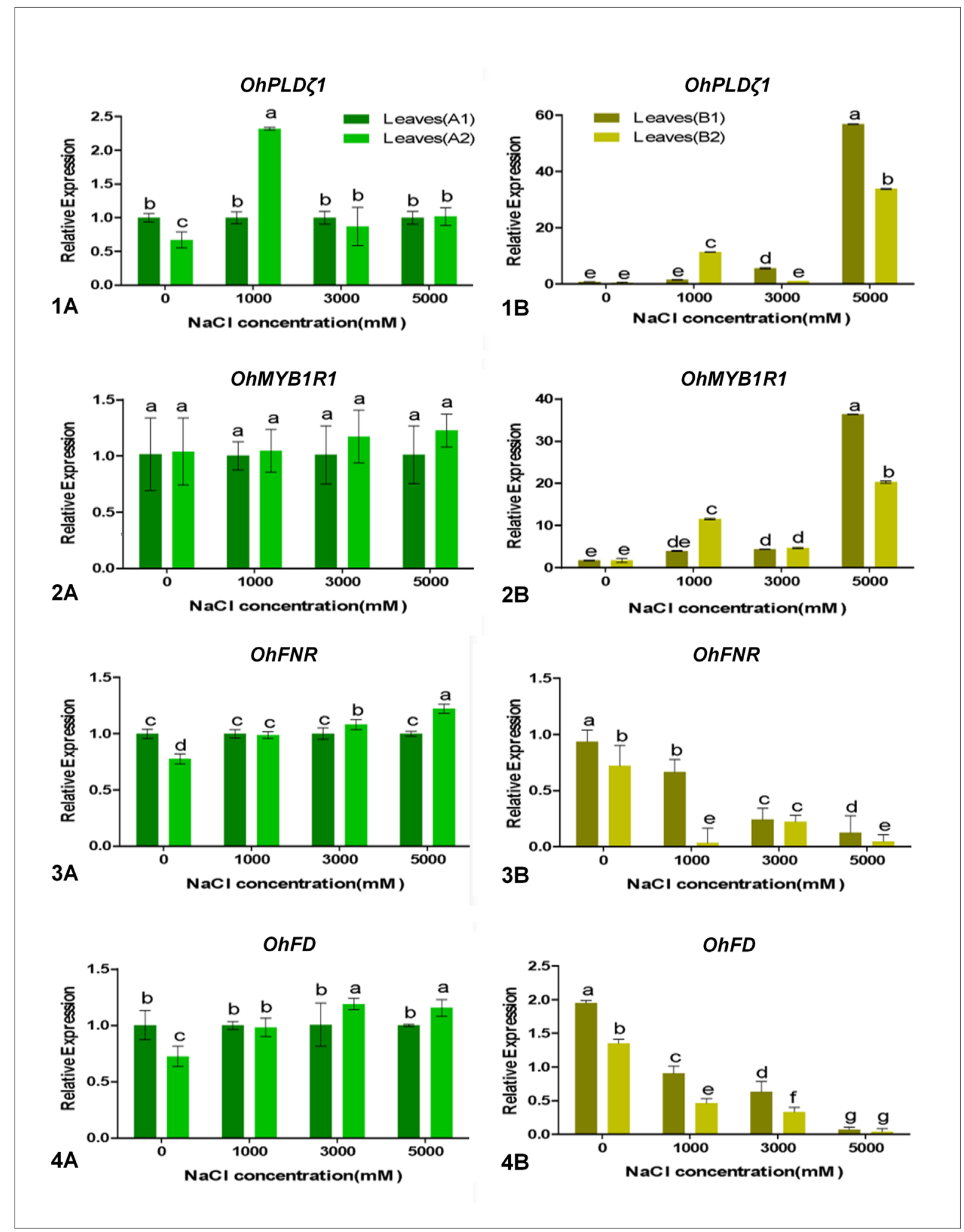

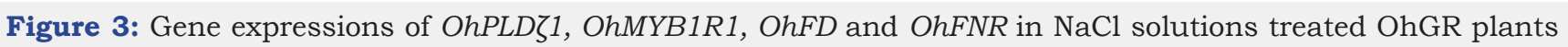
probed by qPCR.

1A-4A: Upper leaves.

1B-4B: Basal leaves. 


\section{Discussion and Conclusion}

All orchids are listed in the Convention on International Trade in Endangered Species of Wild Fauna and Flora, CITES [6] and preservation, reproduction and development of orchids are of immediate importance. Development of salt-tolerant orchids or even halophytic varieties is a fascinating prospective in future seawater horticulture, providing great possibilities for sustainable development on the saline soil and seashore.

The present work reports salt tolerance in OhGR plants that are never considered or recorded as a kind of halotolerant plant. To verify if the salt tolerance is due to prevention of the salt via absorption, electroconductivities are measured and show that $\mathrm{NaCl}$ is in did penetrated into the inner tissues of the salt treated plants. The salt pre-treatment work shows the salt tolerance cannot be induced or enhanced by low $[\mathrm{NaCl}]$ solutions, i.e. the salt is accumulated in the plant and leaves degenerate when salt concentration passes a certain limit.

The expression of the electron transfer carrier gene OhFD with its coupled carrier gene OhFNR [7] showed a stress induced coinhibition pattern, indicating a progressive power-supply housekeeping disorder. Both OhMYB1R1 and OhPLD 1 [8] are stress response genes at early stages of response [4]. The expression of OhMYB1R1 was found inducible in OhGR plants at lower salt concentration stress condition [4], and the present work showed that it was more enhanced by severe salt stresses. The OhPLD 1 at the present work showed a co-expression pattern of OhMYB1R1 that points to similar responsive position in OhGR response to salt stresses.

The upper leaves, the pseudobulbs and the roots kept morphologically normal and alive in most tested OhGR plants in various salt conditions tested, even a high amount of salt had been accumulated in the organs as revealed by electroconductivity measures. Our results show that OhGR plants have high salt tolerance potential based on their ordered cell and molecular mechanisms.

The investigated degeneration caused by salt treatment was in the 4-leaf stage of the OhGR plant development, and further analysis should be made to check the salt stress effects on whole plant life including e.g. seedling and florescence stages. Even though, the high tolerance of OhGR plants to such high salt concentration, i.e. several times of seawater $[\mathrm{NaCl}]$, already is the potential to explore in orchid breeding and cultivation.

\section{Funding}

This research was funded by Minjiang Scholarshipof Fujian Province (MJJZ13-003), Construction of Plateau Discipline of Fujian Province (Grant No. 102/71201801101) and Sci-Tech Innovation Foundation of Fujian Agriculture and Forestry University (CXZX2017189, CXZX2018076).

\section{Declaration of Competing Interest}

The authors declare that they have no competing financial interests or personal relationships that could have appeared to influence the work reported in this paper.

\section{References}

1. XuHan X, Guo R, Zhang Y, Li R, Xiao X, et al. (2020) Plant halotolerance: evolution and halogenomics. Chinese Journal of Tropical Crops 41(10) :1979-1989.

2. Gong Z, Xiong L, Shi H, Yang S, Herrera Estrella LR, et al. (2020) Plant abiotic stress response and nutrient use efficiency. Science China Life Sciences 63(5): 635-674.

3. Jing X, Hu F (2018) Research progress of Orchidaceae. Molecular Plant Breeding 16(15): 5080-5092.

4. Li R, Wu X, Wang X, Chen Y, Guo R, et al. (2018) Cloning and subcellular localization of RFNR and the mechanisms of stress induced response of RFNR and LFNR in Oncidium. Acta Horticulturae Sinica 45(11): 21642176.

5. Wu X (2017) Optimization of Oncidium in vitro culture system and transformation analysis of ferredoxin genes [M.D. Dissertation]. Fuzhou: Fujian Agriculture and Forestry University, China.

6. (2007) Convention on International Trade in Endangered Species of Wild Fauna and Flora. CITES, pp. 1178-1182.

7. Hanke GT, Kurisu G, Kusunoki M, Hase T (2004) Fd: FNR electron transfer complexes: evolutionary refinement of structural interactions. Photosynthesis Research 81(3): 317-327.

8. Eliáš M, Potocký M, Cvrčková F, Žárský V (2002) Molecular diversity of phospholipase D in angiosperms. BMC Genomics 3: 2. https://doi. org/10.1186/1471-2164-3-2. 